\title{
Left-right symmetry at LHC and precise 1-loop low energy data
}

\author{
J. Chakrabortty, ${ }^{a}$ J. Gluza, ${ }^{b}$ R. Sevillano ${ }^{b}$ and R. Szafron ${ }^{b}$ \\ ${ }^{a}$ Theoretical Physics Department, Physical Research Laboratory, \\ Navarangpura, Ahmedabad 380009, India \\ ${ }^{b}$ Department of Field Theory and Particle Physics, Institute of Physics, \\ University of Silesia, Uniwersytecka 4, PL-40-007 Katowice, Poland \\ E-mail: joydeep@prl.res.in, gluza@us.edu.pl, robert.szafron@us.edu.pl, \\ radomir.sevillano-borkowski@us.edu.pl
}

Abstract: Despite many tests, even the Minimal Manifest Left-Right Symmetric Model (MLRSM) has never been ultimately confirmed or falsified. LHC gives a new possibility to test directly the most conservative version of left-right symmetric models at so far not reachable energy scales. If we take into account precise limits on the model which come from low energy processes, like the muon decay, possible LHC signals are strongly limited through the correlations of parameters among heavy neutrinos, heavy gauge bosons and heavy Higgs particles. To illustrate the situation in the context of LHC, we consider the "golden" process $p p \rightarrow e^{+} N$. For instance, in a case of degenerate heavy neutrinos and heavy Higgs masses at $15 \mathrm{TeV}$ (in agreement with FCNC bounds) we get $\sigma\left(p p \rightarrow e^{+} N\right)>10 \mathrm{fb}$ at $\sqrt{s}=14 \mathrm{TeV}$ which is consistent with muon decay data for a very limited $W_{2}$ masses in the range $(3008 \mathrm{GeV}, 3040 \mathrm{GeV})$. Without restrictions coming from the muon data, $W_{2}$ masses would be in the range $(1.0 \mathrm{TeV}, 3.5 \mathrm{TeV})$. Influence of heavy Higgs particles themselves on the considered LHC process is negligible (the same is true for the light, SM neutral Higgs scalar analog). In the paper decay modes of the right-handed heavy gauge bosons and heavy neutrinos are also discussed. Both scenarios with typical see-saw lightheavy neutrino mixings and the mixings which are independent of heavy neutrino masses are considered. In the second case heavy neutrino decays to the heavy charged gauge bosons not necessarily dominate over decay modes which include only light, SM-like particles.

KeYwords: Beyond Standard Model, GUT

ArXiv EPRINT: 1204.0736 


\section{Contents}

1 Introduction 1

2 One-loop low energy constraints on the right sector in MLRSM 3

3 Consequences of low energy constraints for MLRSM signals at LHC $\quad 6$

3.1 Decay widths and branching ratios of the heavy LR spectrum 6

$\begin{array}{lll}3.2 & \text { LR signals at LHC, a sample } & 9\end{array}$

4 Conclusions

\section{Introduction}

In general there are two ways in which non-standard models can be tested. In the first approach, Standard Model (discovered) processes or observables can be calculated very accurately by taking into account radiative corrections of the non-standard model. In the second approach we can look into completely new effects (new processes) which are not present in the Standard Model (SM) but exist in its extensions. Their detections would be a clear signal for the non-standard physics. Here radiative corrections beyond leading order are, at least at first approximation, not necessary.

At the LHC era it is interesting to think closer how these two approaches could be joined and how we can profit from this situation. It is not a common strategy, especially as Grand Unified Theories (GUT) are concerned. Here we calculate 1-loop radiative corrections at low energies consistently in the framework of the non-standard model (not only in its SM subset, this issue of consistency has been explored intensively in [1-3], see also [4-6]). In the next step we are looking into some specific non-standard process at LHC, taking into account obtained earlier precise low energy predictions for parameters of the model.

We consider left-right symmetric model based on the $\mathrm{SU}(2)_{L} \otimes \mathrm{SU}(2)_{R} \otimes \mathrm{U}(1)_{B-L}$ gauge group [7-13] in its most restricted form, so-called Minimal Left-Right Symmetric Model $(M L R S M)$. We choose to explore the most popular version of the model with a Higgs representation with a bidoublet $\Phi$ and two (left and right) triplets $\Delta_{L, R}[14,15]$. We also assume that the vacuum expectation value of the left-handed triplet $\Delta_{L}$ vanishes, $\left\langle\Delta_{L}\right\rangle=0$ and the CP symmetry can be violated by complex phases in the quark and lepton mixing matrices. Left and right gauge couplings are chosen to be equal, $g_{L}=g_{R}$. For reasons discussed in [1] and more extensively in [16], we discuss see-saw diagonal light-heavy neutrino mixings. It means that $W_{1}$ couples mainly to light neutrinos, while $W_{2}$ couples to the heavy ones. $Z_{1}$ and $Z_{2}$ turn out to couple to both of them $[17,18]$. $W_{L}-W_{R}$ mixing is neglected here. ${ }^{1}$

\footnotetext{
${ }^{1}$ As an interesting detail, the most stringent data comes from astrophysics through the supernova explosion analysis [19, 20], $\xi<3 \cdot 10^{-5}$, typically $\xi<0.05[21]$.
} 
Taking such a restricted model, easier its parametrization and less extra parameters are involved in phenomenological studies. However, it does not mean that it is easier to confirm or falsify it, in fact, despite of many interesting studies and constraints, the model has not been ruled out so far (though many interesting questions and problems calling for consistency of the model have been arose [1-3]. PDG [21] gives $M_{W_{2}}>1 \mathrm{TeV}$ for standard couplings decaying to $e \nu$, recently the CMS collaboration established the generic bound [22] $M_{W_{2}}>1.4 \mathrm{TeV}$. Moreover, CMS published exclusion limits for LR model [23], they excluded large region in parameter space $\left(M_{N}, M_{W_{2}}\right)$ which extends up to $M_{W_{2}}=1.7 \mathrm{TeV}$. Similarly, ATLAS collaboration gives exclusion limits on both $M_{N}$ and $M_{W_{2}}$. They obtained that $M_{W_{2}}>1.8 \mathrm{TeV}$ for difference in mass of $M_{N}$ and $M_{W_{2}}$ larger than $0.3 \mathrm{TeV}$ [24] (for $34 p b^{-1}$ ). The very last ATLAS analysis [25] based on the integrated luminosity of $2.1 \mathrm{fb}^{-1}$ pushed it even further, for some neutrino mass ranges it reaches already $2.3 \mathrm{TeV}$. These exclusion searches assume generally that $M_{W_{2}}>M_{N}$, however in LR model the situation can be different i.e. $M_{W_{2}}<M_{N}$. Let us note that $K_{L}-K_{S}$ data gives for the minimal LR model a strong theoretical limit, which is (at least) at the level of $2.5 \mathrm{TeV}[26,27]$.

In further studies we take then the rough $K_{L}-K_{S}$ limit for $W_{2}$ mass (to which the LHC analysis approaches quickly, and rather sooner than later will overcome it)

$$
M_{W_{2}}>2.5 \mathrm{TeV} \text {. }
$$

For heavy neutrino limit $M_{N}>780 \mathrm{GeV}$ [24], but it must be kept in mind that bounds on $M_{N}$ and $M_{W_{2}}$ are not independent from each other. Let us mention that simultaneous fit to low energy charge and neutral currents give $M_{W_{2}}>715 \mathrm{GeV}[21,29]$.

Neutrinoless double beta decay allows for heavy neutrinos with relatively light masses, if eq. (1.1) holds, for more detailed studies, see e.g. [27, 28].

Detailed studies which take into account potential signals with $\sqrt{s}=14 \mathrm{TeV}$ at LHC conclude that heavy gauge bosons and neutrinos can be found with up to 4 and $1 \mathrm{TeV}$, respectively, for typical LR scenarios [30-32]. Anyway, such a relatively low (TeV) scale of the heavy sector is theoretically possible, even if GUT gauge unification is demanded, for a discussion, see e.g. [33] and [34].

As far as one loop corrections are concerned, there are not many papers devoted to the LR model. Apart from [1-3, 18] in which one of the authors of this paper has been involved (MLRSM model), there are other papers: [35] (limits on $W_{2}$ mass coming from the $K_{L}-K_{S}$ process (finite box diagrams, renormalization not required), [36, 37] (LEP physics), [38-42] (process $b \rightarrow s \gamma$ ). Some interesting results are included also in papers [43, 44] where the problem of decoupling of heavy scalar particles in low energy processes has been discussed.

On the other hand, the LHC collider gives us a new opportunity to investigate LR models and to look for possible direct signals. Lately a few interesting papers analysed possible signals connected with the LR model [27, 30, 32, 45-48]. As we are looking for nonstandard signals, we restrict here calculations at high energies to the first approximation (tree level).

In the next section we will discuss low energy limits on right sector of MLRSM which come from precise calculation of the muon decay. In section 3 some representative LR 
signals at LHC will be discussed, taking into account severe limits coming from the muon decay analysis. We end up with conclusions. We have decided to skip most of the details connected with definition of fields, interactions and parameters in the MLRSM. All these details can be found in [18] and [2] (especially the appendix there).

\section{One-loop low energy constraints on the right sector in MLRSM}

Four-fermion interactions describe low energy processes in the limit $\frac{q^{2}}{M^{2}} \ll 1$, where $q$ is the transfer of four momentum and $M$ is the mass of the gauge boson involved in the interactions. This is an effective approximation of the fundamental gauge theory. This construction allows to replace the complete interaction by the point interaction with the effective coupling constant (which depends on the model). Independently, the model can be postulated with universal constant coupling (e.g. Fermi model with universal constant $\left.G_{F}\right)$. Next, taking into account the perturbation, corrections to so defined constants can be calculated at higher levels. Both effective and universal procedures describe the same process, so the corrections calculated in this way must be the same. This fact can be used to constrain parameters of the tested model.

In the $\mathrm{SM}$, all radiative corrections are embedded in the $\Delta r$ term [21]

$$
\frac{G_{F}}{\sqrt{2}}=\frac{e^{2}}{8\left(1-M_{W}^{2} / M_{Z}^{2}\right) M_{W}^{2}}(1+\Delta r) .
$$

With the present values of the coupling constants and masses [21]

$$
\begin{aligned}
G_{F} & =1.166364(5) \cdot 10^{-5} \mathrm{GeV}^{-2}, & & 1 / \alpha=137.0359976 \pm 0.00000050, \\
M_{W} & =80.399 \pm 0.023 \mathrm{GeV}, & & M_{Z}=91.1876 \pm 0.0021 \mathrm{GeV},
\end{aligned}
$$

experimental fits to the $\Delta r$ parameter in SM give $[21]^{2}$

$$
\Delta r \equiv \Delta r_{0} \pm \Delta r_{\sigma}=0.0362 \pm 0.0006 .
$$

Matching for the muon decay and the structure of $\Delta r$ in the MLRSM model at the 1-loop level has been discussed in [2], see also [49] for more details on the matching in the context of SM.

In figure $1 \Delta r$ as function of $\mathrm{v}_{R}$ for different masses of heavy Higgs particles and heavy neutrinos is shown. While plotting we have considered the variations of $\Delta r$ with respect to $\mathrm{v}_{R}$, as the heavy gauge boson masses are directly proportional to this parameter,

$$
M_{W_{2}} \simeq 0.47 \mathrm{v}_{R}, \quad M_{Z_{2}} \simeq 0.78 \mathrm{v}_{R}
$$

see figure 5 in [2]. Mass of the lightest neutral Higgs scalar is assumed to be $M_{H_{0}^{0}}=120 \mathrm{GeV}$ ( $\Delta r$ is not sensitive to this mass, see figure 6 in [2]). Masses of remaining heavy Higgs particles $H \equiv\left\{H_{1}^{0}, H_{2}^{0}, H_{3}^{0}, A_{1}^{0}, A_{2}^{0}, H_{1}^{+}, H_{2}^{+}, \delta_{L}^{++} \equiv H_{1}^{++}, \delta_{R}^{++} \equiv H_{2}^{++}\right\}$

$$
M_{H} \equiv M_{H_{1}^{0}}=M_{H_{3}^{0}}=M_{A_{1}^{0}}=M_{A_{2}^{0}}=M_{H_{1}^{+}}=M_{H_{2}^{+}}=M_{H_{1}}^{++}=M_{H_{2}^{0}}=M_{H_{2}}^{++}
$$

\footnotetext{
${ }^{2}$ The error has decreased about 3 times during last decade or so, mostly due to improvements in $W$ boson mass measurement.
} 


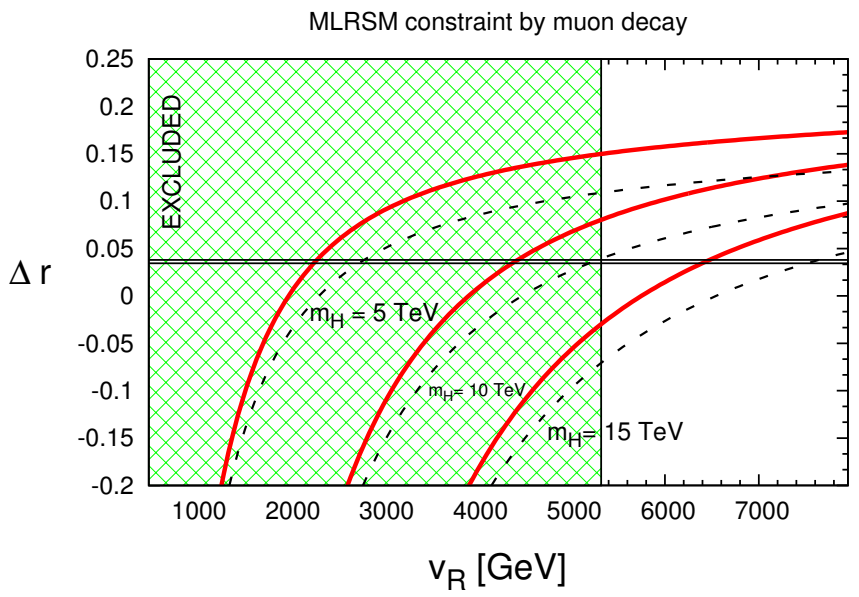

Figure 1. $\Delta r$ as function of $\mathrm{v}_{R}$ for different masses of heavy Higgs particles $5 \mathrm{TeV}, 10 \mathrm{TeV}$ and $15 \mathrm{TeV}$, see eqs. (2.5), (2.6). Solid (dashed) lines are for neutrino heavy masses with $h_{M}=0.1$ $\left(h_{M}=1\right)$, see eq. (2.7). Bold horizontal lines show the $3 \sigma$ C.L. constraint on $\Delta r$, see eq. (2.3). Excluded region comes from the bound on $W_{2}$, eq. (1.1).

are assumed to be equal,

$$
m_{H}=\mathrm{v}_{R}
$$

Heavy neutrino masses

$$
M_{N}=\sqrt{2} h_{M} \mathrm{v}_{R}
$$

are taken in the range $h_{M} \in[0.1,1] . h_{M}$ is the Yukawa coupling connected with the right-handed Higgs triplet. $h_{M}<0.1$ are not forbidden, however attention should be paid to the limits coming from direct experimental searches (LEP $Z_{1}$ decays, ATLAS, CMS), especially for a region of small $\mathrm{v}_{R}$ which we explore. On the other hand, $h_{M}>1$ reaches non-perturbative region.

We can see, as expected in the framework of GUT models to which MLRSM belongs, that for given $m_{H}$ and $M_{N}$ there is a very narrow space for $\mathrm{v}_{R}$ which are consistent with muon data (fine-tuning).

Table 1 describes the situation more precisely. Set $\mathbf{A}$ shows ranges of $\mathrm{v}_{R}$ which fit at $3 \sigma$ C.L. to eq. (2.3) for varying heavy neutrino masses in the range $h_{M} \in[0.1,1]$, see eq. (2.7). The upper limit of $\mathrm{v}_{R}$ corresponds to neutrino masses with $h_{M}=1$ and $\Delta r_{\max }=$ $\Delta r_{0}+3 \Delta r_{\sigma}$, the lower limit of $\mathrm{v}_{R}$ corresponds to $h_{M}=0.1$ and $\Delta r_{\min }=\Delta r_{0}-3 \Delta r_{\sigma}$, see figure 2. We can see that the heavy degenerate neutrinos can be relatively light having masses below $1 \mathrm{TeV}$. A minimal heavy neutrino mass for $\left(\mathrm{v}_{R}\right)_{\min }$ depicted with asterisk in the last column could be even smaller (if $h_{M}<0.1$ ). For instance, $\mathrm{v}_{R}=6398 \mathrm{GeV}$ $\left(M_{W_{2}} \simeq 3 \mathrm{TeV}\right)$ and muon data in the range $\Delta r_{0} \pm 3 \Delta r_{\sigma}$ restricts allowed heavy neutrino masses to the region $100 \leq M_{N} \leq 2210$ [in GeV] (it means that $\left(h_{M}\right)_{\min } \simeq 0.01$ ). Set B describes a range of $M_{N}$ which fits at $3 \sigma$ C.L. to eq. (2.3) where in addition also $\mathrm{v}_{R}$ is fixed. Here a fixed point is chosen to be a value of $\mathrm{v}_{R}$ which for given $m_{H}$ and a neutrino mass with $h_{M}=1$ gives $\Delta r_{\min }=\Delta r_{0}-3 \Delta r_{\sigma}$ (crossing with lower of horizontal lines in 


\begin{tabular}{|c|c|c|c|c|}
\hline set $\mathbf{A}$ & $m_{H}=4 \mathrm{TeV}$ & $m_{H}=5 \mathrm{TeV}$ & $m_{H}=10 \mathrm{TeV}$ & $m_{H}=15 \mathrm{TeV}$ \\
\hline $\mathrm{v}_{R}[\mathrm{GeV}]$ & $(1809,2263)$ & $(2257,2795)$ & $(4373,5283)$ & $\left(6398^{*}, 7639\right)$ \\
$M_{N}[\mathrm{GeV}]$ & $(256,3200)$ & $(319,3952)$ & $(618,7471)$ & $(905,10803)$ \\
\hline set $\mathbf{B}$ & $m_{H}=4 \mathrm{TeV}$ & $m_{H}=5 \mathrm{TeV}$ & $m_{H}=10 \mathrm{TeV}$ & $m_{H}=15 \mathrm{TeV}$ \\
& $\mathrm{v}_{R}=2214 \mathrm{GeV}$ & $\mathrm{v}_{R}=2738 \mathrm{GeV}$ & $\mathrm{v}_{R}=5189 \mathrm{GeV}$ & $\mathrm{v}_{R}=7513 \mathrm{GeV}$ \\
$M_{N}[\mathrm{GeV}]$ & $(2974,3131)$ & $(3717,3872)$ & $(6974,7336)$ & $(10118,10623)$ \\
\hline
\end{tabular}

Table 1. Set A. Values of $\mathrm{v}_{R}$ for which various Higgs masses give $\Delta r$ in agreement with eq. (2.3). The ranges of $v_{R}$ are achieved by varying heavy neutrino masses in the domain $h_{M} \in[0.1,1]$, see eq. (2.7). For $\left(\mathrm{v}_{R}\right)_{\min }$ depicted by an asterisk in the last column, and corresponding $\left(M_{N}\right)_{\min }$ for which $h_{M}<0.1$, see the main text.

Set B. $v_{R}$ is fixed in addition, leaving as the only free MLRSM parameter the neutrino mass $M_{N}$, see figure 2. Values obtained for $m_{H} \leq 5 \mathrm{TeV}$ do not fulfil direct LHC experimental search limits, the same is true for $m_{H}=10 \mathrm{TeV}$ if the limit eq. (1.1) is applied.

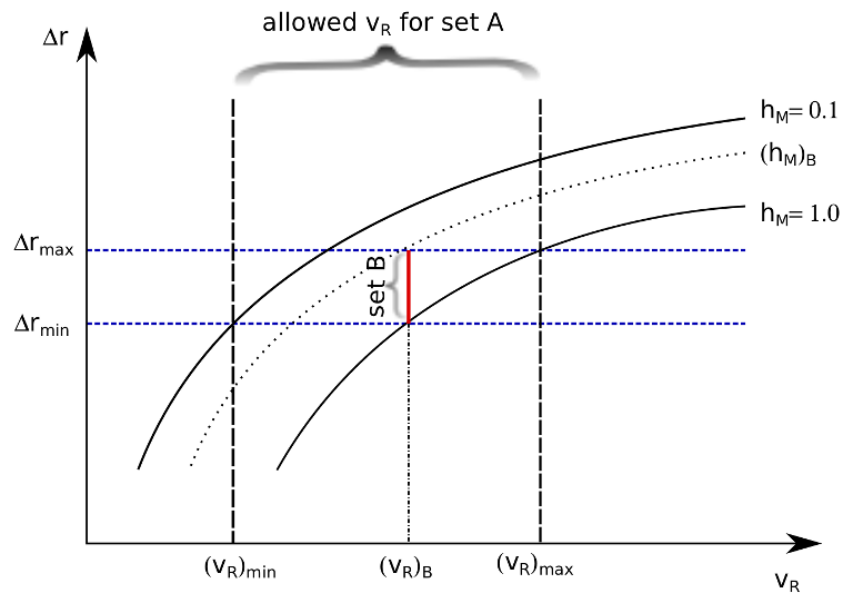

Figure 2. Scheme for limited parameters in table 1. $\left(\mathrm{v}_{R}\right)_{B}$ is a fixed value of $\mathrm{v}_{R}$ for which set $\mathrm{B}$ is defined with maximal value of degenerate heavy neutrino mass (in the perturbative region, $\left.h_{M}=1\right)$.

figure 1). Then we are looking for $h_{M}<1$ which still covers $3 \sigma$ C.L. region constraint by eq. (2.3) and we get the range of neutrino masses written in the table 1 , see figure 2 .

For Set B possible values of $M_{N}$ are of course even more restricted than for Set $\mathbf{A}$.

Results in table 1 are compatible with eq. (1.1) for the last column, $m_{H}=15 \mathrm{TeV}$. If we take into account FCNC, neutral heavy Higgs mass should be larger than $10-15 \mathrm{TeV}$, going down to a few $\mathrm{TeV}$ only in some special cases (for references and update discussion, see [50]). So, from now on, let us focus on the last column, $m_{H}=15 \mathrm{TeV}$. If we start with some other value of $\mathrm{v}_{R}$ instead $\left(\mathrm{v}_{R}\right)_{B}$, e.g. $\mathrm{v}_{R}=6500 \mathrm{GeV}\left(M_{W_{2}} \simeq 3055 \mathrm{GeV}\right)$ and muon data in the range $\Delta r_{0} \pm 3 \Delta r_{\sigma}$ restricts allowed heavy neutrino masses to the region $2654 \leq M_{N} \leq 3232 \mathrm{GeV}$. 


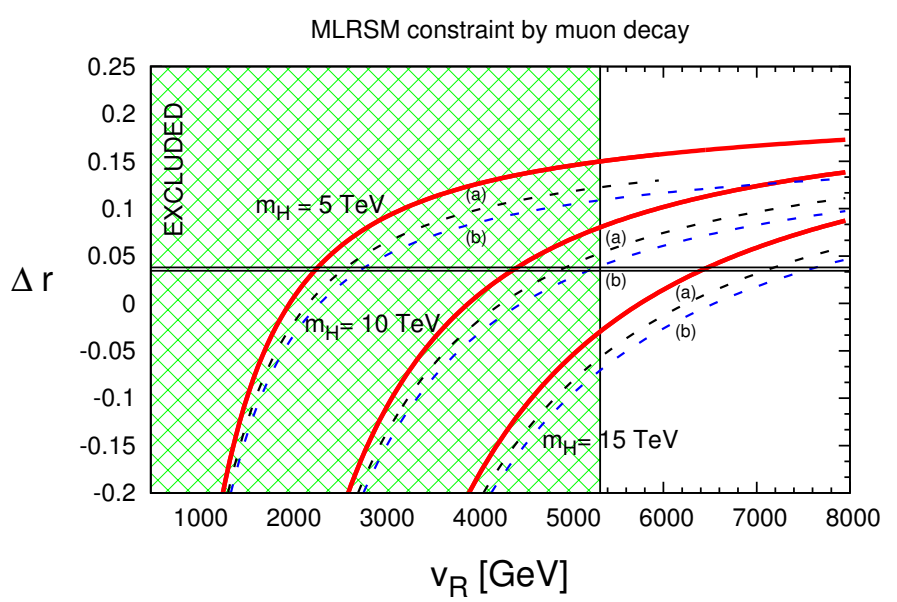

Figure 3. $\Delta r$ as function of $\mathrm{v}_{R}$ for three different masses of heavy Higgs particles, $5 \mathrm{TeV}, 10 \mathrm{TeV}$ and $15 \mathrm{TeV}$. Cases (a) and (b) are different by the heavy neutrino mass spectrum. In the case (a) a mass of $N_{4}$ is fixed, $M_{N_{4}}=800 \mathrm{GeV}$ and masses of $N_{5}, N_{6}$ neutrinos vary with $\mathrm{v}_{R}$, eq. (2.7). In the case (b) all $N_{4}, N_{5}, N_{6}$ neutrinos have masses which obey eq. (2.7). For the solid lines with $h_{M}=0.1$ the neutrino cases (a) and (b) give the same predictions. Bold horizontal lines show the $3 \sigma$ C.L. constraint on $\Delta r$, see eq. (2.3).

To discuss a case with non-degenerate neutrinos, in figure 3 we let one of the heavy neutrinos to be much lighter, $M_{N_{4}}=800 \mathrm{GeV}$ (for $N_{5}, N_{6}$ we keep masses through the relation eq. (2.7)). We call it the case (a). For the case (b) we vary all three heavy masses with $\mathrm{v}_{R}$, in accordance with eq. (2.7) (degeneracy, the same $h_{M}$ ). In the case $h_{M}=0.1$ there is only one line, as two cases (a) and (b) give the same predictions. We can see that lines change slightly with chosen neutrino mass spectrum, but not dramatically, values of allowed $\mathrm{v}_{R}$ are relatively stable and well constrained.

In summary, heavy $\left(m_{H}>10 \mathrm{TeV}\right)$ Higgs masses are allowed and follow roughly $\mathrm{v}_{R}$ scale (allowed $\mathrm{v}_{R}$ increases with increasing $m_{H}$ ). However, the most important for the LHC phenomenology is the fact that still light (at the level of hundreds of $\mathrm{GeV}$ ) heavy neutrinos are allowed in the framework of MLRSM. Let us discuss it more carefully.

\section{Consequences of low energy constraints for MLRSM signals at LHC}

\subsection{Decay widths and branching ratios of the heavy LR spectrum}

Experimental limits on $W_{L}-W_{R}$ mixing angle $\xi$ are very severe and, similarly as in the muon decay case, we neglect it here. Second, as already mentioned in Introduction, we assume MLRSM with diagonal light-heavy neutrino mixings of the "see-saw" type

$$
\left|U_{\nu_{i} j}\right| \simeq \frac{\left|\left\langle M_{D}\right\rangle\right|}{M_{N_{j}}} \delta_{i, j-3}, \quad i=1,2,3, \quad j=4,5,6
$$

where $\left\langle M_{D}\right\rangle$ is an order of magnitude of the Dirac neutrino mass matrix and $\nu_{i}$ stands for 3 light neutrinos.

These two are conservative assumptions, on the other hand they are very natural and we can see what signals we can get at LHC for such harsh model conditions. For instance, 

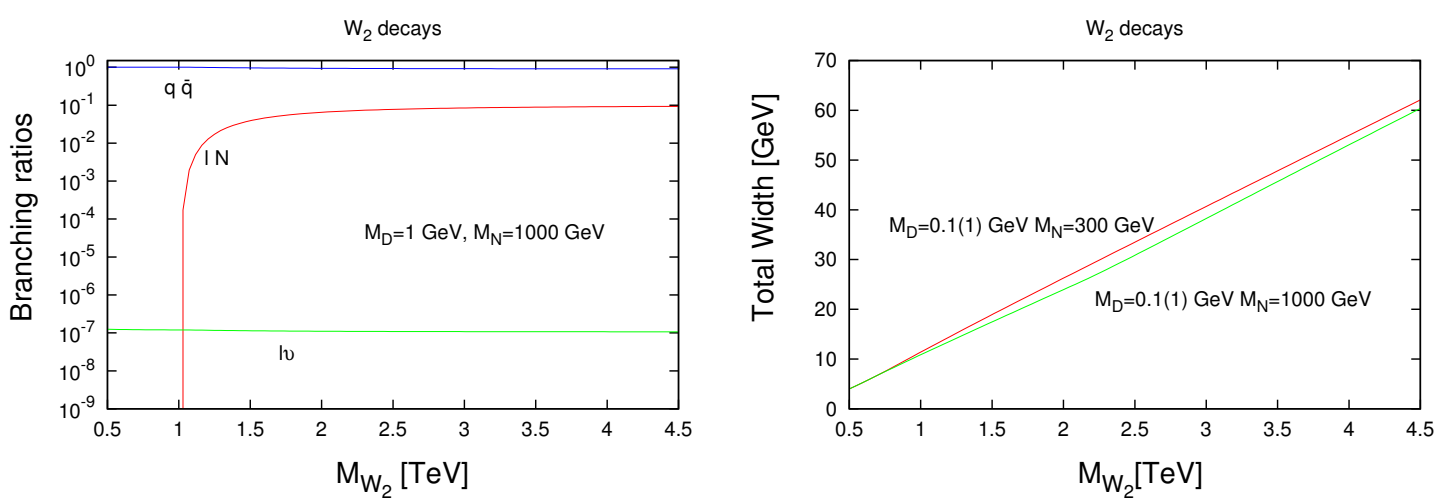

Figure 4. Decay branching fractions and total widths for $W_{2}$ decays. Symbol $q \bar{q}$ on this and next plots stands for a sum of all quark flavours, $q \bar{q} \equiv \sum_{i, i^{\prime}=u, d, s, b, c, t} q_{i} \overline{q_{i^{\prime}}}$. Similarly, $l N \equiv \sum_{i=4}^{6} l_{i-3} N_{i}, l \nu \equiv \sum_{i=1}^{3} l_{i} \nu_{i}$

analyzed in [53] signals which stem from the gauge boson triple vertices including heavy gauge bosons are absent completely in our scenario.

In figure 4 we can see that heavy gauge boson decay is dominated by quark channels. ${ }^{3}$ Second of importance is $W_{2}$ decay to heavy neutrinos, that is why these two channels make the "golden" process considered in the next section large. As the mixing in eq. (3.1) becomes smaller, the $l \nu$ decay mode falls, e.g. for $M_{D}=0.1 \mathrm{GeV}$ we obtain $\operatorname{Br}\left(W_{2} \rightarrow l \nu\right) \simeq 10^{-11}$. These are a kind of textbook results, see e.g. [54] and references therein.

However, there are scenarios in which branching ratios can be different and heavy particles can decay dominantly to the light particles, so not through the right-handed currents. This is a case of non see-saw models where mixing angles are independent of heavy neutrino masses, see e.g. [16].

Let us assume then that light-heavy neutrino mixing defined in eq. (3.1) is independent of the heavy neutrino mass, experimental limits on elements of this mixing read (this limit has improved substantially over the last decade) [57]

$$
\sum_{j=4,5,6} U_{\nu_{1}, j-3} U_{\nu_{1}, j-3}^{*}=U_{\nu_{1}, 4} U_{\nu_{1}, 4}^{*} \leq 0.003 \equiv \kappa_{\max }^{2} .
$$

In this case, the $l \nu$ branching ratio in figure 4 will enhance, ${ }^{4} \mathrm{BR}\left(W_{2} \rightarrow l \nu=5 \cdot 10^{-4}\right)$. Still, it is not large. $q \bar{q}$ and $l N$ modes dominate.

In figure 5 decays of the $Z_{2}$ boson are shown. Also here results are practically independent of light-heavy mixing scenarios, eqs. (3.1), (3.2). $Z_{2}$ heavy boson decays are also dominated by quark channels. Here the situation is more complicated and up to a per mil level, a few channels contribute. Interestingly, also $Z_{2}$ decay to a pair of light neutrinos or bosons as well as to the $Z_{1} H_{0}^{0}$ pair are substantial.

\footnotetext{
${ }^{3}$ In figure 4 and the next we do not depict explicitly exclusion regions (e.g. eq. (1.1)), as the limits for the heavy particle spectrum change quickly with increasing LHC luminosity, see e.g. [24] vs. [25].

${ }^{4}$ In a case where more than one heavy neutrino state exists (which is true in MLRSM), the maximal light-heavy neutrino mixing defined in eq. (3.2) is constrained further among others by neutrinoless double beta decay measurements to be less than $\kappa_{\max }^{2} / 2$ [55]. We take then this parameter in our considerations for non-decoupling light-heavy neutrino mixings.
} 

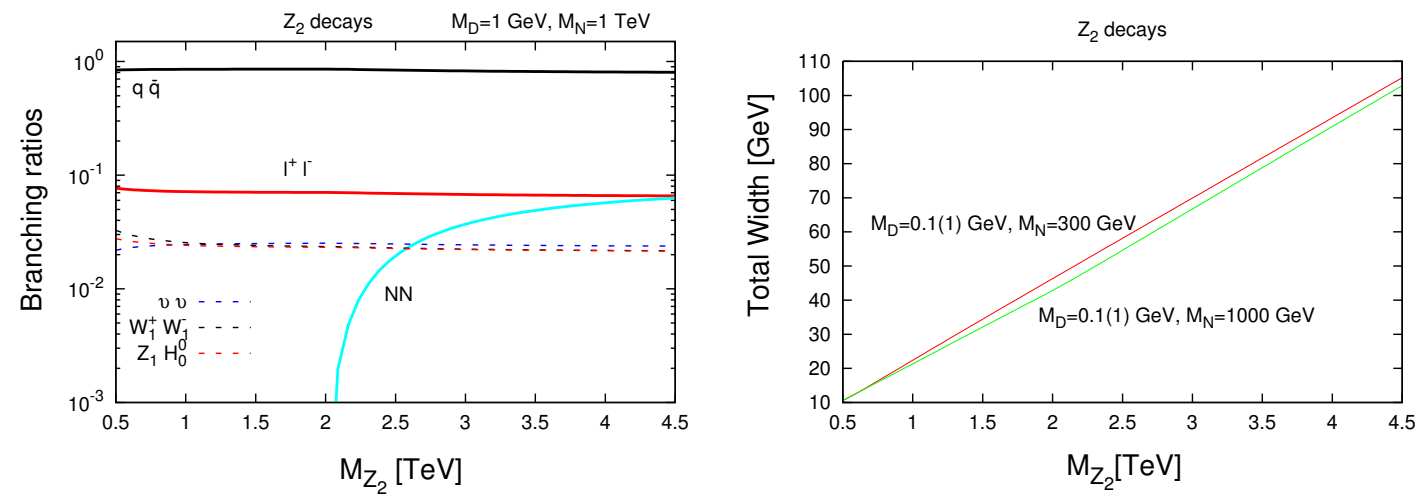

Figure 5. Decay branching fractions and total widths for $Z_{2}$ decays.
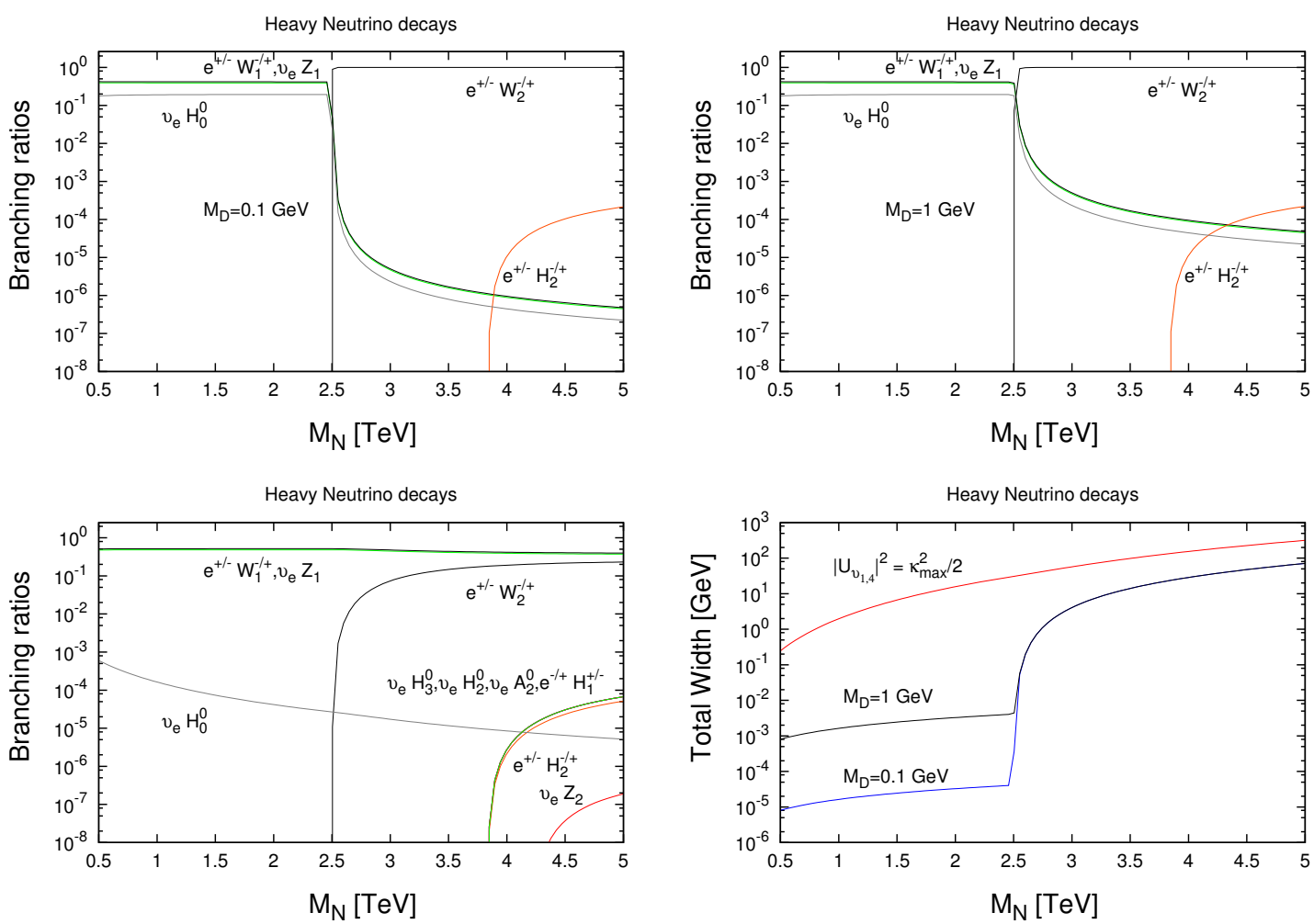

Figure 6. Decay branching fractions and total widths for heavy neutrino decays with see-saw type of mixing, eq. (3.1), first row $\left(\left\langle M_{D}\right\rangle=0.1 \mathrm{GeV}\right.$ (on left) and $1 \mathrm{GeV}$ (on right)). In the second row, on left, branching ratios with maximal type of mixings are calculated, eq. (3.2). On right the total widths are given. $M_{W_{2}}$ is fixed at $2.5 \mathrm{TeV}$.

However, the situation changes with respect to light-heavy mixing scenarios for the case of heavy neutrino decays. Decays of the first of heavy neutrinos $N_{4}$ in figure 6 are dominated by the $e^{ \pm} W_{1}^{\mp}$ (we neglect here the mixing between different generations) mode till the threshold where $W_{2}$ production is open. Mass of $W_{2}$ is fixed at $2.5 \mathrm{TeV}$. Still $e^{ \pm} W_{1}^{\mp}$ option is large, even if $W_{2}$ mass would be smaller $\left(1.5 \mathrm{TeV} \leq M_{W_{2}} \leq 2.5 \mathrm{TeV}\right)$. Changing the mixing eq. (3.1) affects mainly $\nu_{e} Z_{2}$ mode (which is negligible). 


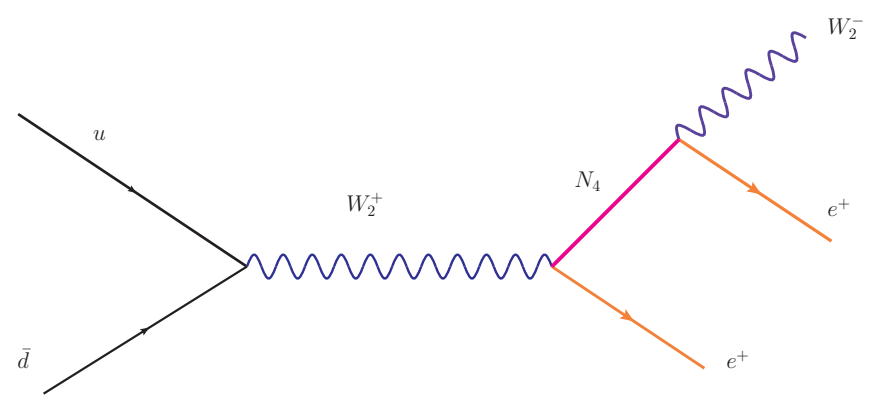

Figure 7. A tree level basic diagram for the $u \bar{d} \rightarrow e^{+} e^{+} W_{2}^{-}$process. The process is not suppressed if $W_{2}^{+}$decays to right-handed quarks forming jets (it is suppressed if it decays to standard, lefthanded leptons).

If we took maximal possible mixing, $\kappa_{\max }^{2} / 2=0.0015$, then the branching ratios for heavy neutrino decays change qualitatively (left figure in the second row in figure 6). We can see that the $N_{4} \rightarrow e W_{1}$ and $N_{4} \rightarrow \nu Z_{1}$ decays dominate over decay channels to the heavy states $W_{2}, Z_{2}$ in the kinematically allowed regions. The reason is that although decay amplitudes for light boson modes are proportional to the small light-heavy neutrino mixing, the helicity summed amplitudes for gauge boson modes are suppressed in addition by the masses of gauge bosons, which is a stronger effect in a case of heavy gauge bosons. The difference between both scenarios of neutrino mixings is clearly visible on the last plot in figure 6 where total decay widths are given.

In order to show influence of the Higgs sector we deliberately distorted heavy Higgs mass spectrum to include some lighter Higgs masses such that Higgs particles show up in the neutrino decay. However, open in this way Higgs decay modes contribute well below per mille level in total and are negligible.

\subsection{LR signals at LHC, a sample}

The so-called "golden" process where the left-right symmetry signal is not suppressed due to small light-heavy neutrino mixings is depicted in figure 7 (here heavy neutrino couples directly to $W_{2}$ which decays hadronically, figure 4 ). Thus the final state consists of the same sign di-leptons and jets which also carries a clear signature of lepton number violation. Even if we consider the leptonic decay modes of $W_{2}$, we can have 3-leptons and missing energy as our signal events. The presence of one missing energy source allows to reconstruct $W_{2}$ fully, and then the reconstruction of the right-handed neutrino, $N_{i}$, helps to reduce the combinatorial backgrounds for this process. In [27] it has been discussed that the dominant background for this process is coming from $t \bar{t}$ events and is negligible beyond the $\mathrm{TeV}$ scale. In the other case where $W_{2}$ decays hadronically with the largest branching fraction, the invariant mass of the hardest jets plus one(two) lepton(s) also allows to reconstruct in a clean way the heavy neutrino $N$ and $W_{2}$ masses.

As discussed in the last section, muon decay data restricts very much possible values of $\mathrm{v}_{R}$ (and through the relation eq. (2.4) masses of heavy gauge bosons) for chosen spectrum of Higgs and neutrino masses. Let us then assume a scenario for LHC potential discoveries 


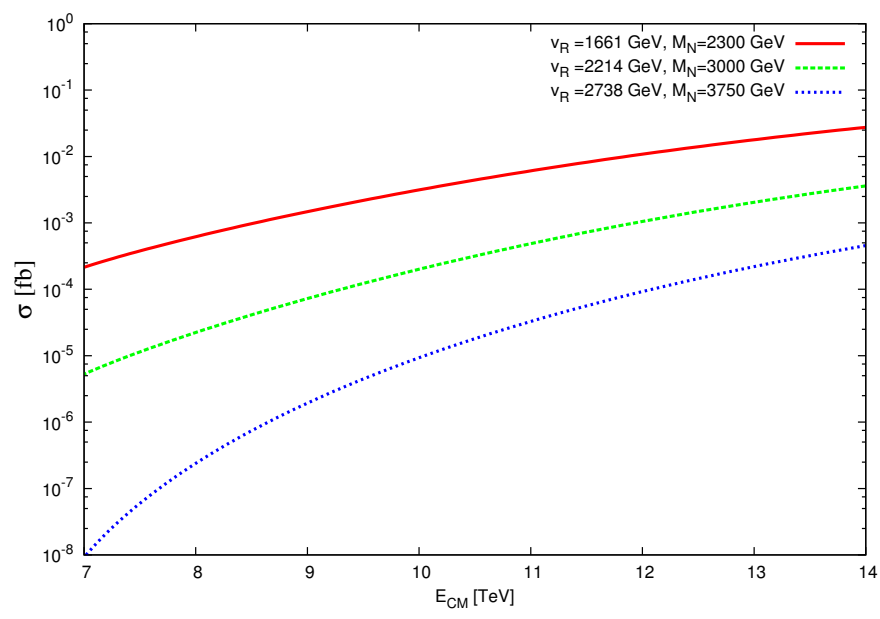

Figure 8. Cross-sections for processes $p p \rightarrow e^{ \pm} N_{4} \rightarrow e^{ \pm} e^{ \pm} W_{2}^{\mp}$ for sets of parameters in Set B, $m_{H}=3,4,5 \mathrm{TeV}$, respectively.

with $M_{W_{2}} \simeq 2.5 \mathrm{TeV}$ (then $\mathrm{v}_{R} \simeq 5 \mathrm{TeV}$ ). If we choose the most uniform scenario defined by Set $\mathbf{B}$ in table 1 (with the same masses for all Higgs particles and also for all heavy neutrinos), then muon decay data sets the heavy neutrino masses of the order $7 \mathrm{TeV}$ (and masses of Higgs particles of the order of $10 \mathrm{TeV}$ ).

We have computed the cross-section ${ }^{5}$ for the process $p p \rightarrow e^{ \pm} N_{4} \rightarrow e^{ \pm} e^{ \pm} W_{2}^{\mp}$ for the sets of parameters given in Set B (table $1, v_{R}=1661 \mathrm{GeV}$ is for $m_{H}=3 \mathrm{TeV}$ ). Signatures for heavy neutrinos and charged gauge bosons in hadron colliders have been discussed already some decades ago, for a first paper on these kind of signals, see [56]. Results are shown in figure 8. As can be seen in this case the cross-section is very small for LHC operating at $7 \mathrm{TeV}$, results for higher $m_{H}$ will give even smaller values. Going to $14 \mathrm{TeV}$ of course improve the situation but still this scenario is very unlikely to be discovered.

Luckily, other scenarios are possible where one of heavy right-handed neutrinos has smaller mass, e.g. $800 \mathrm{GeV}$, but other two are very heavy having masses $\sim 5 \mathrm{TeV}$. There is also an option with 3 degenerate heavy neutrinos but with smaller $\mathrm{v}_{R}$, e.g. $\mathrm{v}_{R}=6398 \mathrm{GeV}$ $\left(M_{W_{2}} \simeq 3 \mathrm{TeV}\right)$, see the last column in table 1 . These scenarios are still compatible with muon decay data (though relatively light heavy gauge boson is required). It gives much bigger cross-section, see figure 9 , with anticipated luminosity this is a detectable process.

From the above plot it is clear that as the mass of the heavy neutrino and the scale $\mathrm{v}_{R}$ increase, the production cross-section falls rapidly and then the further decays of the $N$ followed by the decay of $W_{2}$ suppress the effective cross-section for this "golden" process.

However, in figure 10 we show more carefully how precise low energy data from table 1 restricts a space of possible cross-section for this process. Let us assume that Higgs masses are degenerate, at the level of $10 \mathrm{TeV}$ and $15 \mathrm{TeV}$ (the first case is almost ex-

\footnotetext{
${ }^{5}$ For numerical results we use CalcHEP [58] and Madgraph5 [59] with our own implementation of the MLRSM model in Feynrules [60]. We made a couple of cross checks for correctness of implementations for neutrino and gauge boson mixings. Results for $e^{-} e^{+} \rightarrow \nu N$ [17], $e^{-} e^{-} \rightarrow W_{1}^{-} W_{1}^{-}$[51, 52], $e^{-} \gamma \rightarrow$ $N W_{1}^{-}[55]$ and $p p \rightarrow l W_{2}[32]$ have been recovered, among others.
} 


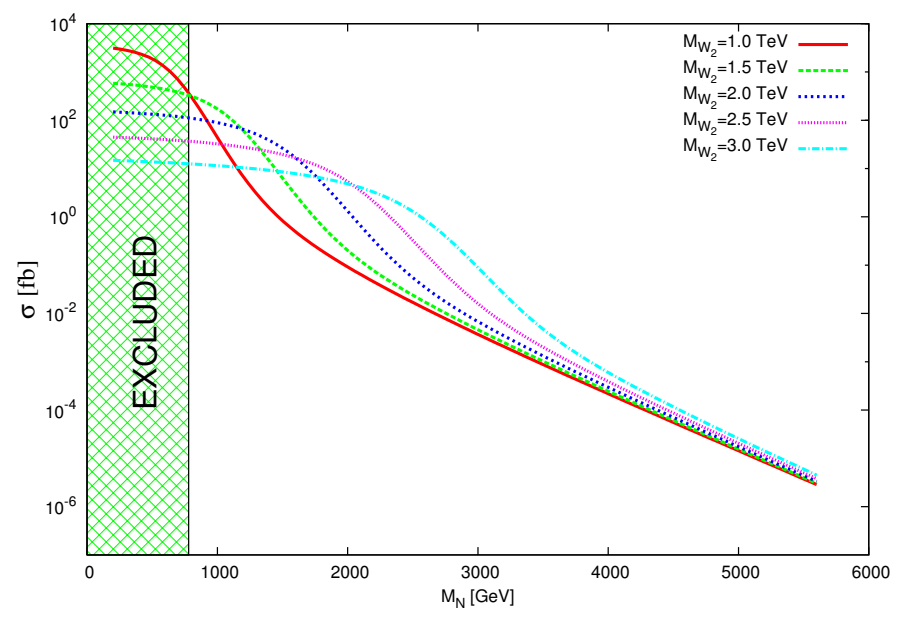

Figure 9. Cross-section for process $p p \longrightarrow e^{+} N_{4}$ as a function of heavy neutrino mass $M_{N}$ for different sets of $W_{2}$ masses, $\sqrt{s}=14 \mathrm{TeV}$. Excluded region of $M_{N}$ depends on $M_{W_{2}}$, see the plots in $[24,25]$. We have just fixed it safely at $M_{N}=780 \mathrm{GeV}$.

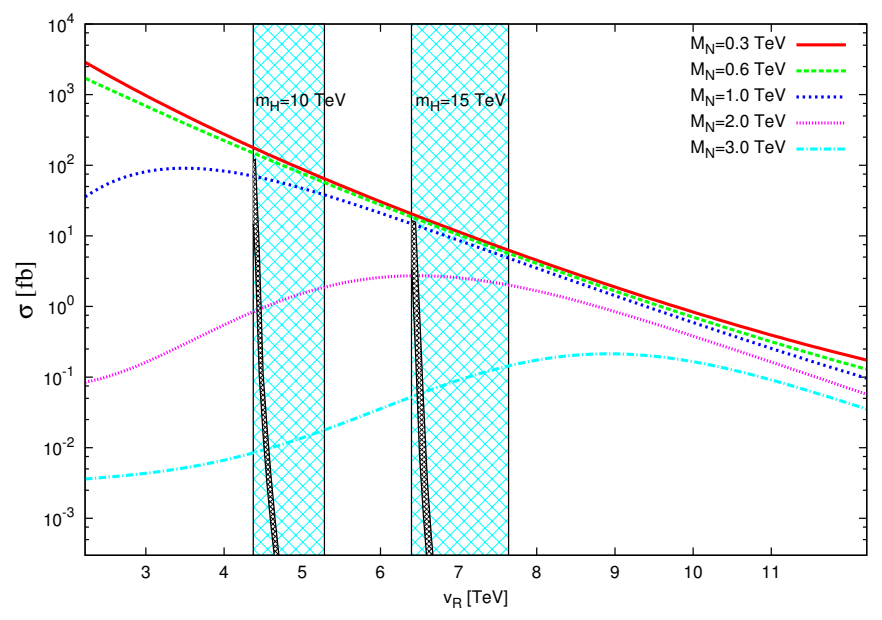

Figure 10. Cross-section for process $p p \longrightarrow e^{+} N_{4}$ for different sets of heavy neutrino masses $M_{N}, \sqrt{s}=14 \mathrm{TeV}$. The results are for degenerate heavy neutrino and Higgs particle masses. $m_{H}$ masses are fixed at $10 \mathrm{TeV}$ and $m_{H}=15 \mathrm{TeV}$. The whole shaded bands correspond to parameters labeled as Set $\mathbf{A}$ in table 1 and figure 2. In addition, for each $\mathrm{v}_{R}$ between $\left(\mathrm{v}_{R}\right)_{\min }$ and $\left(\mathrm{v}_{R}\right)_{B}$ in figure 2, heavy neutrino mass spectrum which is in agreement with muon decay data is obtained (we call it Set B). In this way a possible cross-section for allowed $M_{W_{2}}-M_{N}$ masses is constrained dramatically. These regions are denoted by almost vertical and thin black stripes within the wider shaded regions.

cluded, see eqs. (1.1), (2.7) and table 1). Then vertical bands restrict regions of possible cross-sections for given $M_{N}$ masses. If we assume in addition that heavy neutrino masses are also degenerate, then the black, thin strips inside these bands give for each $\mathrm{v}_{R}$ very narrow intervals of possible heavy neutrino masses, consequently, region of possible crosssections is very limited. With an assumed luminosity of tens of inverse femtobarns at 
$\sqrt{s}=14 \mathrm{TeV}, \sigma\left(p p \rightarrow e^{+} N\right) \simeq 10 \mathrm{fb}$ would give hundreds of events, which we take as a safety discovery limit for this process. Relevant experimental conditions do not spoil signals, for a discussion on kinematical cuts and a background for this process, see e.g. [32]. In this case, without muon data, possible $\mathrm{v}_{R}$ values give $W_{2}$ mass in the range $(1 \mathrm{TeV}$, $3.5 \mathrm{TeV}$ ) for heavy neutrino masses up to $1 \mathrm{TeV}$. Muon data shrinks the region very much, $1970 \mathrm{GeV} \leq M_{W_{2}} \leq 2050 \mathrm{GeV}$ (for $m_{H}=10 \mathrm{TeV}$ ) and $3008 \mathrm{GeV} \leq M_{W_{2}} \leq 3040 \mathrm{GeV}$ (for $m_{H}=15 \mathrm{TeV}$ ). From figure 10 it should be clear that increasing heavy Higgs masses would shift the $v_{R}$ scale to higher level, decreasing further cross sections for the considered process. In summary, for the left-right LHC phenomenology, Higgs mass spectrum is optimal in vicinity of $15 \mathrm{TeV}$ region. For a case of degenerate heavy neutrinos, heavy Higgs particles with masses at about $10 \mathrm{TeV}$ and below are practically excluded by muon data. On the other hand, MLRSM scenarios with Higgs particles masses at about $20 \mathrm{TeV}$ (and above) are allowed by muon data, however, low energy muon restrictions constraint heavy gauge boson and neutrino masses in such a way that $\sigma\left(p p \rightarrow e^{+} N\right)<10 \mathrm{fb}$.

Let us stress that there is no problem in the considered model to get heavy Higgs particle masses at the level of $15 \mathrm{TeV}$ (or above) while keeping the lightest Higgs particle to be at the level of $125 \mathrm{GeV}$. Their masses are proportional to the $v_{R}$ and $\kappa_{1,2}$ VEVs, respectively $[14,15,18]$.

In this paper we do not focus on discriminating the LR model from other beyond SM models, which is an important issue by itself and deserves separate detailed studies. A kind of signals considered here (e.g. trilepton plus $E_{T}$ ) can be obtained also in other models, e.g. SUSY, in which $3 \ell$ and $E_{T}$ is an important mode for looking for chargino neutralino production, see e.g. [61, 62]. In general it is not an easy task to distinguish between different models by looking only at the trilepton signal. At the LHC the best but not a trivial way is to look for leptons spin correlations and their angular distributions. This will help to determine spins of intermediate particles. However, the full analysis using all available different channels is needed in order to determine the model which produces the signal; different models must be distinguished by analysing discovered particles, their masses and couplings. The problem of model identification at the LHC in the case of a signal coming from $3 \ell$ and $E_{T}$ was discussed in [63].

\section{Conclusions}

It is very important to take into account low energy data in phenomenological analysis of non-standard models at LHC. This is a quite common action in supersymmetric models, e.g. precise $(g-2)_{\mu}$ analysis is very important for pinning down parameter space for supersymmetry collider searches [64,65]. These kind of analysis are less popular in GUT models (it is justified if a decoupling of heavy states occurs). We should acknowledge the last work [45] where a connection between neutrinoless doubly beta decay and LHC for LR models is undertaken (this is however by its nature purely "tree level" calculation and connection).

Here we show the interplay between fermion-boson heavy spectrum of the MLRSM model in the muon decay. As it is typical for GUT models, it is also true for MLRSM that 
"extensions of the SM in most cases end up in a fine tuning problem, because decoupling of new heavy states, in theories where masses are generated by spontaneous symmetry breaking, is more the exception than the rule" (quotation from [66]). As shown in section 2, fixing heavy gauge boson masses and Higgs particle masses, the region of possible heavy neutrino mass spectrum is restricted by the muon decay. However, there is still a way to get at least one relatively light heavy neutrino, which can be explored at LHC. This is possible as heavy particles effects are effectively "weighted" at the 1-loop level (for virtual particles the effects are summed up which means that effects of 3 heavy degenerate neutrinos can be equivalent to the effects of one relatively light and two heavier heavy neutrino masses). In this case, there is still a way that left-right symmetry is broken at low enough energy scale such that LR models can be discovered directly at LHC (see section 3).

Let us note, that the situation gets more interesting if LHC finds heavy particles which appear in the spectrum of the LR model. Then analysis could be reversed - the obtained physical parameters can be helpful to further pin down remaining parameters for a part of the spectrum which can not be directly constrained at LHC, through the low energy precise analysis like the muon decay. For instance, knowledge of both the mass of the lightest of heavy neutrinos and of the scale $\mathrm{v}_{R}\left(W_{2}\right.$ boson mass reconstruction) will restrict masses of heavier neutrinos in $\Delta r$. This will be a great hint for searches of remaining particles since we would be able to predict where to look for them.

We think that this kind of low-high energy analysis is important and should be further explored, for instance including 1-loop level calculations in MLRSM for lepton flavour violating processes. In general, when making numerical predictions for any model beyond the SM, as many as possible of low energy observables and precision LEP observables should be taken into account.

\section{Acknowledgments}

We would like to thank Henryk Czyż, Fred Jegerlehner, Miha Nemevšek and Marek Zrałek for useful discussions and comments. Work supported by the Research Executive Agency (REA) of the European Union under the Grant Agreement number PITN-GA-2010-264564 (LHCPhenoNet) and by the Polish Ministry of Science under grant No. N N202 064936.

Open Access. This article is distributed under the terms of the Creative Commons Attribution License which permits any use, distribution and reproduction in any medium, provided the original author(s) and source are credited.

\section{References}

[1] M. Czakon, J. Gluza and J. Hejczyk, Muon decay to one loop order in the left-right symmetric model, Nucl. Phys. B 642 (2002) 157 [hep-ph/0205303] [INSPIRE].

[2] M. Czakon, M. Zralek and J. Gluza, Left-right symmetry and heavy particle quantum effects, Nucl. Phys. B 573 (2000) 57 [hep-ph/9906356] [INSPIRE]. 
[3] M. Czakon, J. Gluza, F. Jegerlehner and M. Zralek, Confronting electroweak precision measurements with new physics models, Eur. Phys. J. C 13 (2000) 275 [hep-ph/9909242] [INSPIRE].

[4] M.-C. Chen, S. Dawson and T. Krupovnickas, Constraining new models with precision electroweak data, Int. J. Mod. Phys. A 21 (2006) 4045 [hep-ph/0504286] [InSPIRE].

[5] P.H. Chankowski, S. Pokorski and J. Wagner, (Non)decoupling of the Higgs triplet effects, Eur. Phys. J. C 50 (2007) 919 [hep-ph/0605302] [INSPIRE].

[6] P.H. Chankowski, S. Pokorski and J. Wagner, Z-prime and the Appelquist-Carrazzone decoupling, Eur. Phys. J. C 47 (2006) 187 [hep-ph/0601097] [InSPIRE].

[7] J.C. Pati and A. Salam, Lepton number as the fourth color, Phys. Rev. D 10 (1974) 275 [Erratum ibid. D 11 (1975) 703-703] [INSPIRE].

[8] R. Mohapatra and J.C. Pati, A natural left-right symmetry, Phys. Rev. D 11 (1975) 2558 [INSPIRE].

[9] R.N. Mohapatra and J.C.Pati, Left-right gauge symmetry and an isoconjugate model of CP violation, Phys. Rev. D 11 (1975) 566 [InSPIRE].

[10] G. Senjanović and R.N Mohapatra, Exact left-right symmetry and spontaneous violation of parity, Phys. Rev. D 12 (1975) 1502 [INSPIRE].

[11] R.N. Mohapatra and P.B. Pal, Natural seesaw mechanism, eV-keV-MeV type neutrino spectrum and cosmology, Phys. Rev. D 38 (1988) 2226 [INSPIRE].

[12] G. Senjanović, Spontaneous breakdown of parity in a class of gauge theories, Nucl. Phys. B 153 (1979) 334 [INSPIRE].

[13] H. Georgi and S. Glashow, Unity of all elementary particle forces, Phys. Rev. Lett. 32 (1974) 438 [InSPIRE].

[14] J. Gunion, J. Grifols, A. Mendez, B. Kayser and F.I. Olness, Higgs bosons in left-right symmetric models, Phys. Rev. D 40 (1989) 1546 [inSPIRE].

[15] N. Deshpande, J. Gunion, B. Kayser and F.I. Olness, Left-right symmetric electroweak models with triplet Higgs, Phys. Rev. D 44 (1991) 837 [InSPIRE].

[16] J. Gluza, On teraelectronvolt Majorana neutrinos, Acta Phys. Polon. B 33 (2002) 1735 [hep-ph/0201002] [INSPIRE].

[17] J. Gluza and M. Zralek, Neutrino production in $e^{+} e^{-}$collisions in a left-right symmetric model, Phys. Rev. D 48 (1993) 5093 [INSPIRE].

[18] P. Duka, J. Gluza and M. Zralek, Quantization and renormalization of the manifest left-right symmetric model of electroweak interactions, Annals Phys. 280 (2000) 336 [hep-ph/9910279] [INSPIRE].

[19] R. Barbieri and R.N. Mohapatra, Limits on right-handed interactions from SN1987A observations, Phys. Rev. D 39 (1989) 1229 [INSPIRE].

[20] P. Langacker and S.U. Sankar, Bounds on the mass of $W(R)$ and the $W(L)-W(R)$ mixing angle $\xi$ in general $\mathrm{SU}(2)_{L} \times \mathrm{SU}(2)_{R} \times \mathrm{U}(1)$ models, Phys. Rev. D 40 (1989) 1569 [InSPIRE].

[21] Particle Data Group collaboration, K. Nakamura et al., Review of particle physics, J. Phys. G 37 (2010) 075021 [INSPIRE]. 
[22] CMS collaboration, S. Chatrchyan et al., Search for a $W^{\prime}$ boson decaying to a muon and a neutrino in pp collisions at $\sqrt{s}=7$ TeV, Phys. Lett. B 701 (2011) 160 [arXiv:1103.0030] [INSPIRE].

[23] CMS collaboration, Search for a heavy neutrino and right-handed $W$ of the left-right symmetric model in pp collisions at $\sqrt{s}=7$ TeV, CMS-PAS-EXO-11-002 (2011).

[24] ATLAS collaboration, A search for heavy Majorana neutrino and WR in dilepton plus jets events with the ATLAS detector in pp collisions at $\sqrt{s}=7 \mathrm{TeV}$, ATLAS-CONF-2011-115 (2011).

[25] ATLAS collaboration, G. Aad et al., Search for heavy neutrinos and right-handed $W$ bosons in events with two leptons and jets in pp collisions at $\sqrt{s}=7$ TeV with the ATLAS detector, arXiv: 1203.5420 [INSPIRE].

[26] Y. Zhang, H. An, X. Ji and R.N. Mohapatra, General CP-violation in minimal left-right symmetric model and constraints on the right-handed scale, Nucl. Phys. B 802 (2008) 247 [arXiv:0712.4218] [INSPIRE].

[27] A. Maiezza, M. Nemevšek, F. Nesti and G. Senjanović, Left-right symmetry at LHC, Phys. Rev. D 82 (2010) 055022 [arXiv: 1005.5160] [InSPIRE].

[28] J. Chakrabortty, H.Z. Devi, S. Goswami and S. Patra, Neutrinoless double- $\beta$ decay in TeV scale Left-Right symmetric models, arXiv:1204.2527 [INSPIRE].

[29] M. Czakon, J. Gluza and M. Zralek, Low-energy physics and left-right symmetry: Bounds on the model parameters, Phys. Lett. B 458 (1999) 355 [hep-ph/9904216] [InSPIRE].

[30] M. Nemevšek, F. Nesti, G. Senjanović and Y. Zhang, First limits on left-right symmetry scale from LHC data, Phys. Rev. D 83 (2011) 115014 [arXiv:1103.1627] [INSPIRE].

[31] V. Bansal, ATLAS sensitivity to leptoquarks, $W(R)$ and heavy Majorana neutrinos in final states with high-p $p_{T}$ dileptons and jets with early LHC data at 14 TeV proton-proton collisions, arXiv:0910.2215 [INSPIRE].

[32] A. Ferrari et al., Sensitivity study for new gauge bosons and right-handed Majorana neutrinos in pp collisions at $s=14$ TeV, Phys. Rev. D 62 (2000) 013001 [INSPIRE].

[33] N. Shaban and W.J. Stirling, Minimal left-right symmetry and $\mathrm{SO}(10)$ grand unification using LEP coupling constant measurements, Phys. Lett. B 291 (1992) 281 [INSPIRE].

[34] M. Lindner and M. Weiser, Gauge coupling unification in left-right symmetric models, Phys. Lett. B 383 (1996) 405 [hep-ph/9605353] [INSPIRE].

[35] G. Beall, M. Bander and A. Soni, Constraint on the mass scale of a left-right symmetric electroweak theory from the $K(L) K(S)$ mass difference, Phys. Rev. Lett. 48 (1982) 848 [INSPIRE].

[36] A. Pilaftsis, Confronting left-right symmetric models with electroweak precision data at the $Z$ peak, Phys. Rev. D 52 (1995) 459 [hep-ph/9502330] [InSPIRE].

[37] Z. Gagyi-Palffy, A. Pilaftsis and K. Schilcher, Gauge independent analysis of $K(L) \rightarrow e \mu$ in left-right models, Nucl. Phys. B 513 (1998) 517 [hep-ph/9707517] [INSPIRE].

[38] P. Ball, J. Frere and J. Matias, Anatomy of mixing induced CP asymmetries in left-right symmetric models with spontaneous CP-violation, Nucl. Phys. B 572 (2000) 3 [hep-ph/9910211] [INSPIRE]. 
[39] M. Pospelov, FCNC in left-right symmetric theories and constraints on the right-handed scale, Phys. Rev. D 56 (1997) 259 [hep-ph/9611422] [INSPIRE].

[40] K. Kiers, J. Kolb, J. Lee, A. Soni and G.-H. Wu, Ubiquitous CP-violation in a top inspired left-right model, Phys. Rev. D 66 (2002) 095002 [hep-ph/0205082] [INSPIRE].

[41] T.G. Rizzo, Constraints from $b \rightarrow s \gamma$ on the left-right symmetric model, Phys. Rev. D 50 (1994) 3303 [hep-ph/9401319] [INSPIRE].

[42] P.L. Cho and M. Misiak, $b \rightarrow s \gamma$ decay in $\mathrm{SU}(2)_{L} \times \mathrm{SU}(2)_{R} \times \mathrm{U}(1)$ extensions of the standard model, Phys. Rev. D 49 (1994) 5894 [hep-ph/9310332] [INSPIRE].

[43] G. Senjanović and A. Sokorac, Left-right symmetric gauge theory and its prediction for parity violation in atoms, Phys. Lett. B 76 (1978) 610 [INSPIRE].

[44] G. Senjanović and A. Sokorac, Effects of heavy Higgs scalars at low-energies, Phys. Rev. D 18 (1978) 2708 [inSPIRE].

[45] V. Tello, M. Nemevšek, F. Nesti, G. Senjanović and F. Vissani, Left-right symmetry: from LHC to neutrinoless double beta decay, Phys. Rev. Lett. 106 (2011) 151801 [arXiv: 1011.3522] [INSPIRE].

[46] M. Frank, A. Hayreter and I. Turan, Top quark pair production and asymmetry at the Tevatron and LHC in left-right models, Phys. Rev. D 84 (2011) 114007 [arXiv:1108.0998] [INSPIRE].

[47] M. Frank, A. Hayreter and I. Turan, Production and decays of $W_{R}$ bosons at the LHC, Phys. Rev. D 83 (2011) 035001 [arXiv: 1010.5809] [inSPIRE].

[48] T. Jezo, M. Klasen and I. Schienbein, LHC phenomenology of general $\mathrm{SU}(2) \times \mathrm{SU}(2) \times \mathrm{U}(1)$ models, arXiv: 1203.5314 [INSPIRE].

[49] M. Awramik and M. Czakon, Complete two loop electroweak contributions to the muon lifetime in the standard model, Phys. Lett. B 568 (2003) 48 [hep-ph/0305248] [INSPIRE].

[50] D. Guadagnoli and R.N. Mohapatra, TeV scale left right symmetry and flavor changing neutral Higgs effects, Phys. Lett. B 694 (2011) 386 [arXiv: 1008.1074] [INSPIRE].

[51] J. Gluza and M. Zralek, Inverse neutrinoless double beta decay in gauge theories with CP-violation, Phys. Rev. D 52 (1995) 6238 [hep-ph/9502284] [INSPIRE].

[52] J. Gluza, Constraints on neutrino parameters and doubly charged Higgs particles in the $e^{-} e^{-} \rightarrow W^{-} W^{-}$process, Phys. Lett. B 403 (1997) 304 [hep-ph/9704202] [INSPIRE].

[53] E. Arik et al., A study of $p p \rightarrow W^{\prime} \rightarrow W Z$ at LHC in the ATLAS experiment, ATL-PHYS-2001-005 (2001).

[54] R.N. Mohapatra, Unification and supersymmetry, Springer, U.S.A. (2003).

[55] J. Gluza, J. Maalampi, M. Raidal and M. Zralek, Heavy neutrino mixing and single production at linear collider, Phys. Lett. B 407 (1997) 45 [hep-ph/9703215] [INSPIRE].

[56] W.-Y. Keung and G. Senjanović, Majorana neutrinos and the production of the right-handed charged gauge boson, Phys. Rev. Lett. 50 (1983) 1427 [INSPIRE].

[57] F. del Aguila, J. de Blas and M. Pérez-Victoria, Effects of new leptons in electroweak precision data, Phys. Rev. D 78 (2008) 013010 [arXiv:0803.4008] [INSPIRE].

[58] A. Pukhov, CalcHEP 2.3: MSSM, structure functions, event generation, batchs and generation of matrix elements for other packages, hep-ph/0412191 [INSPIRE]. 
[59] J. Alwall, M. Herquet, F. Maltoni, O. Mattelaer and T. Stelzer, MadGraph 5: going beyond, JHEP 06 (2011) 128 [arXiv:1106.0522] [INSPIRE].

[60] N.D. Christensen and C. Duhr, FeynRules - Feynman rules made easy, Comput. Phys. Commun. 180 (2009) 1614 [arXiv:0806.4194] [INSPIRE].

[61] H. Baer, C.-h. Chen, F. Paige and X. Tata, Trileptons from chargino-neutralino production at the CERN Large Hadron Collider, Phys. Rev. D 50 (1994) 4508 [hep-ph/9404212] [INSPIRE].

[62] Z. Sullivan and E.L. Berger, Trilepton production at the CERN LHC: standard model sources and beyond, Phys. Rev. D 78 (2008) 034030 [arXiv:0805.3720] [INSPIRE].

[63] G. Hallenbeck, M. Perelstein, C. Spethmann, J. Thom and J. Vaughan, Model discrimination with the CMS detector: a case study, Phys. Rev. D 79 (2009) 075024 [arXiv:0812.3135] [INSPIRE].

[64] F. Jegerlehner and A. Nyffeler, The muon g-2, Phys. Rept. 477 (2009) 1 [arXiv:0902.3360] [INSPIRE].

[65] K.A. Olive, Colliders and cosmology, Eur. Phys. J. C 59 (2009) 269 [arXiv:0806.1208] [INSPIRE].

[66] F. Jegerlehner, Comment on $H \rightarrow \gamma \gamma$ and the role of the decoupling theorem and the equivalence theorem, arXiv:1110.0869 [INSPIRE]. 